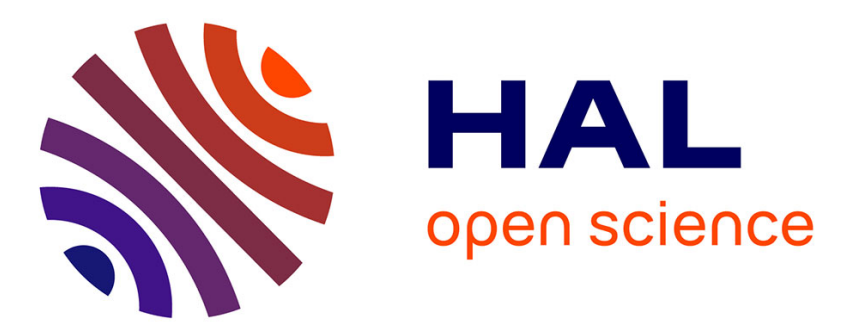

\title{
Evolution of sex hormone binding globulins reveals early gene duplication at the root of vertebrates
}

\author{
Yann Guiguen, Jérémy Pasquier, Alexis Fostier, Julien Bobe
}

\section{To cite this version:}

Yann Guiguen, Jérémy Pasquier, Alexis Fostier, Julien Bobe. Evolution of sex hormone binding globulins reveals early gene duplication at the root of vertebrates. 2020. hal-03048977

\section{HAL Id: hal-03048977 https://hal.inrae.fr/hal-03048977}

Preprint submitted on 9 Dec 2020

HAL is a multi-disciplinary open access archive for the deposit and dissemination of scientific research documents, whether they are published or not. The documents may come from teaching and research institutions in France or abroad, or from public or private research centers.
L'archive ouverte pluridisciplinaire HAL, est destinée au dépôt et à la diffusion de documents scientifiques de niveau recherche, publiés ou non, émanant des établissements d'enseignement et de recherche français ou étrangers, des laboratoires publics ou privés. 


\section{Evolution of sex hormone binding globulins reveals early}

\section{2 gene duplication at the root of vertebrates.}

3 Yann Guiguen ${ }^{1}$, Jeremy Pasquier ${ }^{1}$, Alexis Fostier ${ }^{1}$, Julien Bobe ${ }^{1 *}$

4

$5{ }^{1}$ INRAE, LPGP, 35000 Rennes, France

6 Correspondence to: Julien Bobe, julien.bobe@inrae.fr

7

Highlights:

- Phylogeny, synteny and expression analyses shed new light on Shbg evolution in vertebrates.

- Shbg diversity originates from a duplication event at the root of vertebrate evolution.

- This duplication was followed by many independent losses of Shbg paralogs in vertebrates.

- Shbg paralogs have acquired different tissue expression patterns. 


\section{Abstract}

18 Sex hormone-binding globulin (Shbg) is an important vertebrate blood carrier protein synthetized in the liver and involved in the transport and local regulation of sex steroids in target tissues. A novel shbg gene (shbgb) with a predominant ovarian expression was 21 recently characterized. Being initially found only in salmonids, this shbgb was originally 22 thought to result from the Salmonid-specific whole genome duplication. Using updated transcriptomic and genomic resources we identified Shbgb orthologs in non-salmonid teleosts (European eel, arowana), holosteans (spotted gar, bowfin), polypteriformes (reedfish), agnatha (sea lamprey) and in amphibians, and found that the classical Shbg gene (Shbga) displays a predominant hepatic expression whereas Shbgb has a predominant gonadal expression. Together, these results indicate that these two Shgb genes most likely originate from a whole genome duplication event at the root of vertebrate evolution, followed by numerous and independent losses and by tissue expression specialization of Shbga and Shbgb paralogs.

Keywords: evolution, Sex hormone-binding globulin, teleosts, vertebrates, gene expression

\section{Introduction}

35 Sex hormone-binding globulin (Shbg) is mainly known as a blood-protein carrier involved in

36 the transport of sex steroids in the plasma and in the regulation of their bioavailability to 37 target organs. Shbg proteins present sequence similarities with the other LamG domaincontaining proteins growth arrest-specific 6 (Gas6) and protein S alpha (Pros1) (Joseph, 1997; Joseph and Baker, 1992). By transporting and regulating androgens and estrogens access to the gonads, Shbg plays important roles in vertebrates reproduction (Hammond,

41 2011). The Shbg protein was originally identified in the beta-globulin fraction of the human 42 serum (Rosner et al., 1969) and has previously been known as Androgen-binding protein 
43 (Abp). Shbg genes and Shbg proteins have been characterized in a variety of tetrapod 44 species with the notable exception of birds (Westphal, 1986; Wingfield et al., 1984). In 45 aquatic vertebrates, Shbg was originally found in the plasma of an elasmobranch, the skate 46 (Raja radiata) (Freeman and Idler, 1969), and of a teleost, the rainbow trout (Oncorhynchus 47 mykiss) (Fostier and Breton, 1975). Since that moment on, Shbg have been subsequently 48 identified and studied in many fish species (see for review (Bobe et al., 2010)). Two different shbg genes i.e., shbga and shbgb, have been characterized in teleosts with shbga being the ortholog of the mammalian Shbg, which has been conserved from chondrichthyes to tetrapods and shbgb that has only been reported up to now in salmonids. In contrast to shbga that is mainly expressed in the liver (Bobe et al., 2008), the shbgb transcript was mainly found in the ovary, suggesting a local mediation of the sex steroids effects by the Shbgb protein (Bobe et al., 2008). These two salmonid Shbg proteins share very low identity percentages, with for instance $26 \%$ identity between Shbga and Shgbb at the amino acid level in the rainbow trout (Bobe et al., 2010, 2008). In comparison to other vertebrates and teleost fishes, the salmonid ancestor has experienced an additional (4R) whole genome duplication known as the salmonid specific whole genome duplication, or SaGD (Berthelot et al., 2014). For this reason, and because no shbgb gene had ever been reported in a nonsalmonid species, it was first hypothesized that shbga and shbgb were ohnologous genes resulting from the SaGD (Bobe et al., 2008). This hypothesis was subsequently challenged in another study and the possibility of an ancient duplication followed by a lineage-specific retention in salmonids was suggested (Miguel-Queralt et al., 2009).

The evolutionary history of Shbg genes in vertebrates thus remained unclear and deserved further investigations. Using the increasing amount of genomic and transcriptomic data available for many vertebrate species we revisited the evolutionary history of Shbg genes. The transcriptomes of 24 actinopterygian species (including 22 teleosts) and vertebrate genomes were included in the analysis, which led to the identification of previously noncharacterized Shbgb genes in several non-salmonid vertebrate lineages. Using phylogenomic analyses, we identified several Shbgb orthologs in a variety of non-salmonid 
71 vertebrate species, including teleosts, non-teleost actinopterygians, amphibians and one

72 agnatha. Combined with synteny reconstruction analysis, we demonstrated that Shbg

73 diversity results from a duplication event much older than the SaGD. To gain new information

74 on the functional evolution of shbg genes, we also used quantitative PCR and next

75 generation sequencing approaches, to characterize the expression profiles of shbga and

76 shbgb transcripts for several actinopterygian species. This showed that the paralogous shbg

77 genes have acquired different expression profiles with shbgb having a predominant gonadal

78 expression contrasting with a predominant liver expression of shbga.

\section{Material and Methods}

80

81

82

83

84

85

86

87

88

89

90

91

92

93

94

\subsection{Genomic and transcriptomic databases}

The genomes of the following species, human, Homo sapiens; tropical clawed frog, Xenopus tropicalis; coelacanth, Latimeria chalumnae; spotted gar, Lepisosteus oculatus and zebrafish, Danio rerio were explored using the Ensembl genome browser (http://www.ensembl.org/index.html). The rainbow trout (Oncorhynchus mykiss) genomic database was searched using the Genoscope trout genome browser (http://www.genoscope.cns.fr/trout/). The European eel (Anguilla Anguilla) genomic database was investigated using the European eel assembly available at ZF-Genomics (http://www.zfgenomics.org/sub/eel). Transcriptomes of holostean and teleostean species were investigated using the PhyloFish project resource (Pasquier et al., 2016) available at http://phylofish.sigenae.org. The protein sequences of Human SHBG, Xenopus Shbg, zebrafish Shbg, and rainbow trout Shbga and Shbgb were used as queries to identify homologs of Shbga and Shbgb in the different genomic and transcriptomic databases investigated. A similar methodology was used for Gas6 and Pros1 proteins that were relevant to study due to their phylogenetical proximity and structural similarity. 
95

96

97

98

99

100

101

102

103

104

105

106

107

108

109

110

111

112

113

114

115

116

117

118

119

120

\subsection{Phylogenetic and synteny analyses}

Amino-acid sequences of 126 predicted Shbg (a, b), Gas6 and Pros1 proteins were first aligned using ClustalW (Thompson et al., 1994), then alignments were manually adjusted, to improve the quality of the multiple sequence alignments. The JTT (Jones, Taylor and Thornton) protein substitution matrix of the resulting alignment was determined using ProtTest software (Darriba et al., 2011). Phylogenetic analysis of the proteins presenting LamG domains (i.e. Shbga, Shbgb, Gas6 and Pros1) was performed using the neighbour joining (NJM) method (MEGA 5.1 software), with 1000 bootstrap replicates (Tamura et al., 2011). Trees were edited online with iTOL (Letunic and Bork, 2016) and exported as Scalable Vector Graphics.

Synteny maps of the conserved genomic regions in human, Xenopus, coelacanth, spotted gar and zebrafish were constructed based on information available within the Genomicus (Muffato et al., 2010) v75.01 website (http://www.genomicus.biologie.ens.fr/genomicus75.01/cgi-bin/search.pl). Synteny map of the conserved genomic regions in the rainbow trout was performed using the Rainbow Trout Genomicus Server (http://www.genomicus.biologie.ens.fr/genomicus-trout-01.01/cgi-bin/search.pl). The synteny analyses of European eel conserved genomic regions were obtained performing TBLASTN searches in the corresponding genomic database. For each studied gene, the protein sequences of human and zebrafish were used as queries.

Multiple alignments plots of shbgb genes in salmonids were processed online (http://genome.lbl.gov/vista/) with mVISTA (Dubchak and Ryaboy, 2006; Poliakov et al., 2014) using genomic shbgb sequences of rainbow trout, Oncorhynchus mykiss, Atlantic salmon, Salmon salar, and Coho salmon, Oncorhynchus kisutch. Putative shbgb pseudogenes were retrieved by TBLASTN searches on whole genome sequences using as query the protein sequence of rainbow trout Shbgb. 


\subsection{RNA-seq shbga and shbgb tissue expression in holosteans and teleosts.}

122 RNA-seq and de novo assembly were performed for all studied species as previously 123 described (Berthelot et al., 2014; Braasch et al., 2016; Pasquier et al., 2016). In order to 124 study the expression patterns and levels of shbg transcripts for each actinopterygian species 125 with two shbg genes, we mapped RNA-seq reads on the corresponding shbg coding sequence (CDS) using BWA-Bowtie (Langmead and Salzberg, 2012) with stringent mapping

127 parameters (maximum number of allowed mismatches -aln 2). Mapped reads were counted 128 using SAMtools (Li et al., 2009) idxstat command, with a minimum alignment quality value ($129 \mathrm{q} 30$ ) to discard ambiguous mapping reads. For each species, the numbers of mapped reads 130 were then normalized for each shbg gene across the eleven tissues using the reads per kilo base per million mapped reads (RPKM) normalization. All RNA-seq data are available here: (http://phylofish.sigenae.org/index.html)

\subsection{Quantitative PCR analysis (QPCR).}

QPCR was performed using the RNA collections of the PhyloFish RNA-seq project as previously described (Braasch et al., 2016; Pasquier et al., 2016). Briefly, tissues were sampled from the same female individual and testis from a male individual, when possible. In some species and depending on the tissues, RNA samples from different individuals were pooled to obtain sufficient amounts of RNA. Total RNA was extracted using Tri-Reagent

140 (Molecular Research Center, Cincinnati, OH, USA) according to the manufacturer's instructions. Reverse transcription (RT) was performed using $1 \mu \mathrm{g}$ of RNA for each sample

142 with M-MLV reverse transcriptase and random hexamers (Promega, Madison, WI, USA).

143 Briefly, RNA and dNTPs were denatured for 6 min at $70^{\circ} \mathrm{C}$, chilled on ice for 5 min before the

144 RT master mix was added. RT was performed at $37^{\circ} \mathrm{C}$ for $1 \mathrm{~h}$ and $15 \mathrm{~min}$ followed by a 15 -

145 min incubation step at $70^{\circ} \mathrm{C}$. Control reactions were run without reverse transcriptase and 146 used as negative control in the real-time PCR study. Quantitative RT-PCR (QPCR) 147 experiments were performed sing an Applied Biosystems StepOne Plus. RT products, 
148 including control reactions, were diluted to $1 / 25$, and $4 \mu$ were used for each PCR. All QPCR

149 were performed in triplicates. QPCR was performed using a real-time PCR kit provided with

150 a Fast-SYBR Green fluorophore (Applied Biosystems) with $200 \mathrm{nM}$ of each primer in order to

151 keep PCR efficiency between $80 \%$ and $100 \%$ for all target shbg genes. The relative

152 abundance of target cDNA within a sample set was calculated from serially diluted cDNA

153 pool (standard curve) using Applied Biosystem StepOne V.2.0 software. After amplification, a

154 fusion curve was obtained to validate the amplification of a single PCR product. The fusion

155 curves obtained showed that each primer pair used was specific of a single shbg transcript.

156 The negative control reactions were used to estimate background level. Genes were

157 considered significantly expressed when measured level was significantly above background

158 at $\mathrm{p} \square<\square 0.05$ and within the range of the standard curve. For each studied tissue, cDNA

159 originating from three individual fish were pooled and subsequently used for real-time PCR.

160 Before further analysis, real-time PCR data were collected using the same detection

161 threshold for all studied genes. Data were subsequently normalized using the $\Delta \Delta \mathrm{Ct}$ method

162 to $18 \mathrm{~S}$ transcript abundance in samples diluted to $1: 2,000$.

163

\subsection{Clustering analysis}

165 Expression profiles originating from either QPCR and RNA-seq were represented using supervised clustering methods (Eisen et al., 1998). Hierarchical clustering was processed

167 using centroïd linkage clustering, that uses the average value of all points in a cluster as a 168 reference to calculate distance of other points, with Pearson's uncentered correlation as 169 similarity metric on data that were normalized and median-centered using the Cluster 170 program (Eisen et al., 1998). Results (colorized matrix) of hierarchical clustering analyses 171 were visualized using the Java TreeView program (Saldanha, 2004). 


\section{Results}

\section{$174 \quad 3.1 \quad$ Shbg gene evolution in vertebrates}

175 In order to decipher phylogenetic relationships among Shbg sequences, a phylogenetic 176 reconstruction of the evolution of Shbg was made based using the alignment of 126 177 vertebrate LamG domain-containing proteins. This phylogeny includes Shbg proteins, growth 178 arrest specific 6 proteins (Gas6) and Vitamin K-dependent protein S (Pros1) and the tree 179 was rooted using as outgroup the zebrafish Laminin subunit alpha 4 (lama4). This analysis 180 (Fig. 1) shows that these vertebrate LamG domains proteins cluster into two major clades containing Shbg proteins on one side and the Gas6 and Pros1 proteins on the other side.

182 These Shbg and Gas6/Pros1 clades are both significantly supported with high bootstrap 183 values (i.e. $75 \%$ and $100 \%$, respectively). The Shbg clade contains two sub-clades both 184 supported by significant bootstrap values. The Shbga cluster (100\% bootstrap support), in red, contains all classical vertebrate Shbg proteins from chondrichthyes to teleosts with the notable exception of birds in which no Shbg proteins have been detected (see also Fig.S2).

187 The Shbgb cluster (93\% bootstrap support), in blue, contains not only the salmonid Shbgb 188 proteins, but also other vertebrate sequences outside the salmonid family including sequences from teleosts (European eel and silver arowana), non-teleost bony fishes

190 (reedfish, spotted gar and bowfin), some amphibians and an agnatha, i.e., the sea lamprey 191 (Fig.1 and Fig.S1). The tree topology indicates that Shbgb proteins are not specific to the 192 salmonids lineage and thus suggests a much more ancient origin of Shbgb genes in 193 vertebrates than previously hypothesized (Bobe et al., 2008).

194 To strengthen this phylogeny-based analysis of Shbg protein evolutionary history we carried 195 out a synteny analysis in order to better support this hypothesis of an ancient origin Shbga 196 and Shbgb genes. The synteny analysis first revealed that these two Shbg genes are located 197 on two different syntenic chromosome regions in vertebrates (see Fig. 2A for the Shbga 198 locus and Fig. 2B for the Shbgb locus). In contrast, Gas6 and Pros 1 genes are located on 199 the same syntenic chromosome region in most studied species, with the exception of 
200 primates (Fig. 2C). In vertebrates, Shbga, Shbgb, and Gas6/Pros1 are also located in 201 regions containing other syntenic gene families. These neighboring genes are spread over 202 four syntenic regions (Fig. 2A-2D) like for instance for the Atp-related genes (Atp1b2, 203 Atp1b3, Atp $4 b$ ) that are found in all four syntenic chromosome regions. In addition, Zbtb204 related genes (Ztb4, Zbt38, Zbt33) and Lamp-related genes (Lamp1, Lamp2, Lamp3) are 205 present in three of the four different syntenic chromosome regions depending of the gene 206 family. Altogether, these results strongly suggest that the diversity of the gene family present 207 on these four syntenic chromosome regions probably results from early whole genome 208 duplication events (VG1 and/or VG2) that occurred at the root of vertebrate evolution with a 209 subsequent complex pattern of gene retention and gene losses.

210 Using the recently released salmonid genome resources, we also re-investigated the 211 presence of additional copies of shbg genes in salmonids and confirmed that shbga and 212 shbgb were both retained as single copies (Fig. 1) in rainbow trout, Atlantic salmon and coho 213 salmon suggesting that no functional duplicated copies were retained after the salmonid 214 whole genome duplication (SaGD). However, we also found an additional shbgb gene 215 conserved in these three salmonid species (Fig.3A), but with many stop codons in its 216 deduced open reading frame (see example for rainbow trout in Fig.3B), suggesting that this 217 gene ( $\psi$ shbgb) was subsequently pseudogenized after the SaGD.

\subsection{Expression patterns of shbga and shbgb}

220 In all investigated actinopterygians, shbga was found to be mainly expressed in the liver, 221 supporting a conserved role for this blood-secreted Shbg (Fig.4). However, in the silver arowana a low shbga expression in the gonads is also detected in addition to the predominant liver expression (Fig.4A and Fig.4B). In contrast to shbga, expression of shbgb is not predominant in the liver in all investigated actinopterygians (Fig.4A and Fig.4B). In contrast, shbgb expression is predominantly detected in the gonads (ovary and/or testis) with the notable exception of the European eel. 


\section{Discussion}

228 In this study, we aimed at investigating the diversity of the Shbg family in vertebrates and the evolutionary history of Shbg genes. To date, despite the recent discovery of a second shbg gene (i.e. shbgb) in salmonids, the origin and diversity of Shbg genes in vertebrates has remained controversial. Because salmonids experienced an additional whole genome duplication (SaGD) approximately 100 Mya (Berthelot et al., 2014; Macqueen and Johnston, 2014) compared to other teleost fish and as shbgb genes were initially found in salmonids and, never reported at that time in any non-salmonid species, shbga and shbgb have been first hypothesized to be the result of the SaGD (Bobe et al., 2010, 2008). However pairwise comparison of Shbga and Shbgb reveals a surprisingly low sequence identity (around 25\% at the amino acid level) that was initially interpreted as Shbga and Shbgb being highly divergent SaGD paralog. However, this paralogy relationship was not supported by the phylogeny reconstruction (Bobe et al., 2008) and this discrepancy was thus explained as being the result of a long-branch attraction artifact resulting from the dramatic divergence of these two sequences (Bobe et al., 2010, 2008). Based on the cloning of another salmonid shbgb gene in coho salmon, Oncorhynchus kisutch and the low sequence identity between salmonids

243 Shbga and Shbgb, other authors (Miguel-Queralt et al., 2009), hypothesized that the shbgb gene could stemmed from a much more ancient duplication than the SaGD. In order to decipher the evolutionary history of Shbg genes we re-analyzed the phylogenetic relationships of Shbg genes, their local synteny context, and the evolution of the

247 phylogenetically and structurally closely related genes i.e., Gas6 and Pros 1 that also contain

248 LamG domains and are often identified as potential members of the same family. The 249 identification of new Shbgb genes in vertebrates, the Shbg phylogenetic tree topology and their local synteny relationships strongly suggest that Shbga and Shbgb genes result from a whole genome duplication event that occurred very early at the root on the vertebrate

252 lineage. The presence of a single Shbga gene and a single Shbgb gene in amphibians, 253 holosteans, polypteriformes, agnatha and some teleost fishes, suggests that this Shbg 
254 duplication stems at least from the second round of vertebrate genome duplication (VG2). It 255 is however also possible that Shbga and Shbgb originate the first round of vertebrate 256 genome duplication (VG1) followed by the loss of one duplicate of each gene before VG2.

257 Following this early duplication, these two Shgb paralogs have evolved through many 258 different phylum-specific gene retentions and/or gene losses. Among them the case of birds 259 is interesting as they not only lost their Shbgb gene like reported here for many other 260 tetrapods, but also their Shbga gene that is found to be conserved in all other vertebrates. 261 This complete absence of Shbga in birds has been already reported and it was hypothesized 262 that this specific steroid hormone-binding transport would then be performed by a 263 corticosteroid-binding globulin (Wingfield et al., 1984). Similarly, no Shbg homologs were 264 detected by homology searches in Chondrichthyes (data not shown) but their complete 265 absence in this clade requires further in-depth analysis and additional genome information as 266 Shbg-like sex steroid binding capacities exist in the serum of the Thorny skate (Freeman and 267 Idler, 1969). In tetrapods, Shbgb was only found in Amphibians along with Shbga. Shbgb 268 was also found in holosteans (spotted gar and bowfin), polypteriformes (reedfish), agnatha 269 (sea lamprey) and in a few teleost fish orders i.e., in Elopomorphs (European eel), 270 Osteoglossiforms (silver arowana) and Salmoniforms even though the protein is frequently 271 misannotated in GenBank (Fig.S1). Interestingly we did not find any retention of additional 272 whole genome [SaGD and the teleost specific duplication (TGD)] paralogs for both shbga 273 and shbgb gene with the exception of a pseudogenized SaGD shbgb paralog ( $\psi$ shbgb). This 274 indicates that these extra whole genome duplications did no impact the repertoire of shbg 275 genes with a maximum of one shbga and one shbgb functional copies in all investigated 276 teleost clades. This systematic and independent losses of additional shbga and shbgb 277 duplicated paralogs in teleosts may reflect an evolutionary constraint of maintaining a correct 278 gene and protein dosage as it has been suggested in other organisms (Conant et al., 2014; 279 Gout and Lynch, 2015).

280 In consistency with existing data in mammals, our expression data showed that shbga is 281 predominantly expressed in the liver in the different teleost species studied here. This 
282 confirms what has previously been reported in various teleost species including zebrafish 283 (Danio rerio) (Miguel-Queralt et al., 2004), rainbow trout (Oncorhynchus mykiss) (Bobe et al., 284 2008), Coho salmon (Oncorhynchus kisutch) (Miguel-Queralt et al., 2009), pejerrey 285 (Odontesthes bonariensis) (González et al., 2017) and sea bass (Dicentrarchus labrax) 286 (Miguel-Queralt et al., 2007). In addition, this strong hepatic expression is also observed in 287 spotted gar (Lepisosteus oculatus) and bowfin (Amia calva) as shown by both RNA-seq and 288 QPCR data.

289 In contrast to shbga, data on the tissue distribution of shbgb remain scarce. The ovarian 290 predominant expression of shbgb was originally reported in rainbow trout, in which the 291 transcript could also be detected at lower levels in muscle and stomach (Bobe et al., 2008). 292 Semi quantitative data in Coho salmon confirmed the expression of shbgb in the ovary and 293 stomach and revealed its presence in gills (Miguel-Queralt et al., 2009). Here we show that 294 shbgb is also predominant expressed in the ovary in brown trout, silver arowana and 295 grayling. We also report a strong testicular expression of shbgb in the two holostean species, 296 spotted gar and bowfin, that appears to be lost in teleosts. In addition, the shbgb gene does 297 not exhibit any gonad predominant expression in European eel. Together, our data show that 298 shbga and shbgb have a very specific expression patterns with a predominant expression in 299 liver and gonads, respectively. This pattern appears to be conserved during evolution without 300 any significant change following whole genome duplications events (TGD and SaGD), with 301 the exception of European eel in which the gonad predominant expression of shbgb appears 302 to be lost. Finally, the strong testicular expression of shbgb revealed in bowfin and spotted 303 gar is not found in any teleost species suggesting a specific role of Shbgb in testicular 304 physiology in holostean species.

305

306 The multiple independent losses of Shbgb across vertebrates, while Shbga, Gas6 and Pros 1 307 have been conserved in almost all vertebrates, could reflect different adapative and 308 reproductive strategies as Shbg have been shown to be important carrier proteins for the 309 blood transport of sex steroids and for their delivery to target reproductive tissues 
310 (Hammond, 2011). However, despite this discrepancy among species, the distinct roles of

311 Shgba in hormone transport in the blood and of Shbgb in local hormone action in

312 reproductive organs as well as the associated expression in liver and gonads, respectively, 313 appears to be evolutionary conserved in species that have retained both genes despite a few 314 intriguing species-specific exceptions.

\section{Acknowledgement}

316 This work was supported by the French national research Agency (ANR-10-GENM-017317 PhyloFish).

\section{References}

319 Berthelot, C., Brunet, F., Chalopin, D., Juanchich, A., Bernard, M., Noël, B., Bento, P., Da

Klopp, C., Cabau, C., Gaspin, C., Thorgaard, G.H., Boussaha, M., Quillet, E., Guyomard, R.,

322 Galiana, D., Bobe, J., Volff, J.-N., Genêt, C., Wincker, P., Jaillon, O., Roest Crollius, H.,

323 Guiguen, Y., 2014. The rainbow trout genome provides novel insights into evolution after whole-genome duplication in vertebrates. Nat. Commun. 5, 3657. https://doi.org/10.1038/ncomms4657

Bobe, J., Guiguen, Y., Fostier, A., 2010. Diversity and biological significance of sex hormone-

327 binding globulin in fish, an evolutionary perspective. Mol. Cell. Endocrinol. 316, 66-78.

328 Bobe, J., Mahé, S., Nguyen, T., Rime, H., Vizziano, D., Fostier, A., Guiguen, Y., 2008. A

329 novel, functional, and highly divergent sex hormone-binding globulin that may participate in

330 the local control of ovarian functions in salmonids. Endocrinology 149, 2980-2989.

331 https://doi.org/10.1210/en.2007-1652

332 Braasch, I., Gehrke, A.R., Smith, J.J., Kawasaki, K., Manousaki, T., Pasquier, J., Amores, A., 333 Desvignes, T., Batzel, P., Catchen, J., Berlin, A.M., Campbell, M.S., Barrell, D., Martin, K.J., 334 Mulley, J.F., Ravi, V., Lee, A.P., Nakamura, T., Chalopin, D., Fan, S., Wcisel, D., Cañestro, 
335 C., Sydes, J., Beaudry, F.E.G., Sun, Y., Hertel, J., Beam, M.J., Fasold, M., Ishiyama, M., 336 Johnson, J., Kehr, S., Lara, M., Letaw, J.H., Litman, G.W., Litman, R.T., Mikami, M., Ota, T., 337 Saha, N.R., Williams, L., Stadler, P.F., Wang, H., Taylor, J.S., Fontenot, Q., Ferrara, A., 338 Searle, S.M.J., Aken, B., Yandell, M., Schneider, I., Yoder, J.A., Volff, J.-N., Meyer, A., 339 Amemiya, C.T., Venkatesh, B., Holland, P.W.H., Guiguen, Y., Bobe, J., Shubin, N.H., Di 340 Palma, F., Alföldi, J., Lindblad-Toh, K., Postlethwait, J.H., 2016. The spotted gar genome 341 illuminates vertebrate evolution and facilitates human-teleost comparisons. Nat. Genet. 48, 342 427-437. https://doi.org/10.1038/ng.3526

343 Conant, G.C., Birchler, J.A., Pires, J.C., 2014. Dosage, duplication, and diploidization: 344 clarifying the interplay of multiple models for duplicate gene evolution over time. Curr. Opin.

345 Plant Biol. 19, 91-98. https://doi.org/10.1016/j.pbi.2014.05.008

346 Darriba, D., Taboada, G.L., Doallo, R., Posada, D., 2011. ProtTest 3: fast selection of best-fit 347 models of protein evolution. Bioinforma. Oxf. Engl. 27, 1164-1165.

348 https://doi.org/10.1093/bioinformatics/btr088

349 Dubchak, I., Ryaboy, D.V., 2006. VISTA family of computational tools for comparative 350 analysis of DNA sequences and whole genomes. Methods Mol. Biol. Clifton NJ 338, 69-89. 351 https://doi.org/10.1385/1-59745-097-9:69

352 Eisen, M.B., Spellman, P.T., Brown, P.O., Botstein, D., 1998. Cluster analysis and display of 353 genome-wide expression patterns. Proc. Natl. Acad. Sci. U. S. A. 95, 14863-14868.

354 Fostier, A., Breton, B., 1975. Binding of steroids by plasma of a teleost: the rainbow trout, 355 Salmo gairdneri. J Steroid Biochem.

356 Freeman, H.C., Idler, D.R., 1969. Sex hormone binding proteins. II. Isolation from serum of 357 an elasmobranch (Raja radiata). Gen. Comp. Endocrinol. 13, 83-91.

358 https://doi.org/10.1016/0016-6480(69)90224-x

359 González, A., Fernandino, J.I., Hammond, G.L., Somoza, G.M., 2017. Sex hormone binding 360 globulin: Expression throughout early development and adult pejerrey fish, Odontesthes

361 bonariensis. Gen. Comp. Endocrinol. 247, 205-214.

362 https://doi.org/10.1016/j.ygcen.2017.02.004 
363 Gout, J.-F., Lynch, M., 2015. Maintenance and Loss of Duplicated Genes by Dosage

364 Subfunctionalization. Mol. Biol. Evol. 32, 2141-2148. https://doi.org/10.1093/molbev/msv095

365 Hammond, G.L., 2011. Diverse roles for sex hormone-binding globulin in reproduction. Biol.

366 Reprod. 85, 431-441. https://doi.org/10.1095/biolreprod.111.092593

367 Joseph, D.R., 1997. Sequence and functional relationships between androgen-binding

368 protein/sex hormone-binding globulin and its homologs protein S, Gas6, laminin, and agrin.

369 Steroids 62, 578-588. https://doi.org/10.1016/s0039-128x(97)00045-7

370 Joseph, D.R., Baker, M.E., 1992. Sex hormone-binding globulin, androgen-binding protein,

371 and vitamin K-dependent protein S are homologous to laminin A, merosin, and Drosophila

372 crumbs protein. FASEB J. Off. Publ. Fed. Am. Soc. Exp. Biol. 6, 2477-2481.

373 https://doi.org/10.1096/fasebj.6.7.1532944

374 Langmead, B., Salzberg, S.L., 2012. Fast gapped-read alignment with Bowtie 2. Nat.

375 Methods 9, 357-359. https://doi.org/10.1038/nmeth.1923

376 Letunic, I., Bork, P., 2016. Interactive tree of life (iTOL) v3: an online tool for the display and

377 annotation of phylogenetic and other trees. Nucleic Acids Res. 44, W242-245.

378 https://doi.org/10.1093/nar/gkw290

379 Li, H., Handsaker, B., Wysoker, A., Fennell, T., Ruan, J., Homer, N., Marth, G., Abecasis, G.,

380 Durbin, R., 1000 Genome Project Data Processing Subgroup, 2009. The Sequence

381 Alignment/Map format and SAMtools. Bioinformatics 25, 2078-2079.

382 https://doi.org/10.1093/bioinformatics/btp352

383 Macqueen, D.J., Johnston, I.A., 2014. A well-constrained estimate for the timing of the

384 salmonid whole genome duplication reveals major decoupling from species diversification.

385 Proc. Biol. Sci. 281, 20132881. https://doi.org/10.1098/rspb.2013.2881

386 Miguel-Queralt, S., Blázquez, M., Piferrer, F., Hammond, G.L., 2007. Sex hormone-binding

387 globulin expression in sea bass (Dicentrarchus labrax L.) throughout development and the

388 reproductive season. Mol. Cell. Endocrinol. 276, 55-62.

389 https://doi.org/10.1016/j.mce.2007.06.009

390 Miguel-Queralt, S., Knowlton, M., Avvakumov, G.V., Al-Nouno, R., Kelly, G.M., Hammond, 
391 G.L., 2004. Molecular and functional characterization of sex hormone binding globulin in

392 zebrafish. Endocrinology 145, 5221-5230. https://doi.org/10.1210/en.2004-0678

393 Miguel-Queralt, S., Underhill, C., Devlin, R.H., Hammond, G.L., 2009a. Characterization and

394 measurement of the plasma alpha- and beta-sex hormone-binding globulin paralogs in

395 salmon. Endocrinology 150, 366-375. https://doi.org/10.1210/en.2008-0964

396 Miguel-Queralt, S., Underhill, C., Devlin, R.H., Hammond, G.L., 2009b. Characterization and

397 Measurement of the Plasma $\alpha$ - and $\beta$-Sex Hormone-Binding Globulin Paralogs in Salmon.

398 Endocrinology 150, 366-375. https://doi.org/10.1210/en.2008-0964

399 Muffato, M., Louis, A., Poisnel, C.-E., Roest Crollius, H., 2010. Genomicus: a database and a

400 browser to study gene synteny in modern and ancestral genomes. Bioinforma. Oxf. Engl. 26,

401 1119-1121. https://doi.org/10.1093/bioinformatics/btq079

402 Pasquier, J., Cabau, C., Nguyen, T., Jouanno, E., Severac, D., Braasch, I., Journot, L.,

403 Pontarotti, P., Klopp, C., Postlethwait, J.H., Guiguen, Y., Bobe, J., 2016. Gene evolution and

404 gene expression after whole genome duplication in fish: the PhyloFish database. BMC

405 Genomics 17, 368. https://doi.org/10.1186/s12864-016-2709-z

406 Poliakov, A., Foong, J., Brudno, M., Dubchak, I., 2014. GenomeVISTA--an integrated

407 software package for whole-genome alignment and visualization. Bioinforma. Oxf. Engl. 30,

408 2654-2655. https://doi.org/10.1093/bioinformatics/btu355

409 Rosner, W., Christy, N.P., Kelly, W.G., 1969. Partial purification and preliminary

410 characterization of estrogen-binding globulins from human plasma. Biochemistry $8,3100-$

4113108.

412 Saldanha, A.J., 2004. Java Treeview--extensible visualization of microarray data. Bioinforma.

413 Oxf. Engl. 20, 3246-3248. https://doi.org/10.1093/bioinformatics/bth349

414 Tamura, K., Peterson, D., Peterson, N., Stecher, G., Nei, M., Kumar, S., 2011. MEGA5:

415 molecular evolutionary genetics analysis using maximum likelihood, evolutionary distance,

416 and maximum parsimony methods. Mol. Biol. Evol. 28, 2731-2739.

417 https://doi.org/10.1093/molbev/msr121

418 Thompson, J.D., Higgins, D.G., Gibson, T.J., 1994. CLUSTAL W: improving the sensitivity of 
419 progressive multiple sequence alignment through sequence weighting, position-specific gap

420 penalties and weight matrix choice. Nucleic Acids Res. 22, 4673-4680.

421 Westphal, U., 1986. Steroid-protein interactions II. Monogr. Endocrinol. 27, 1-603.

422 Wingfield, J.C., Matt, K.S., Farner, D.S., 1984. Physiologic properties of steroid hormone-

423 binding proteins in avian blood. Gen. Comp. Endocrinol. 53, 281-292.

424 https://doi.org/10.1016/0016-6480(84)90254-5

425

426

427 


\section{Figures}

429

430

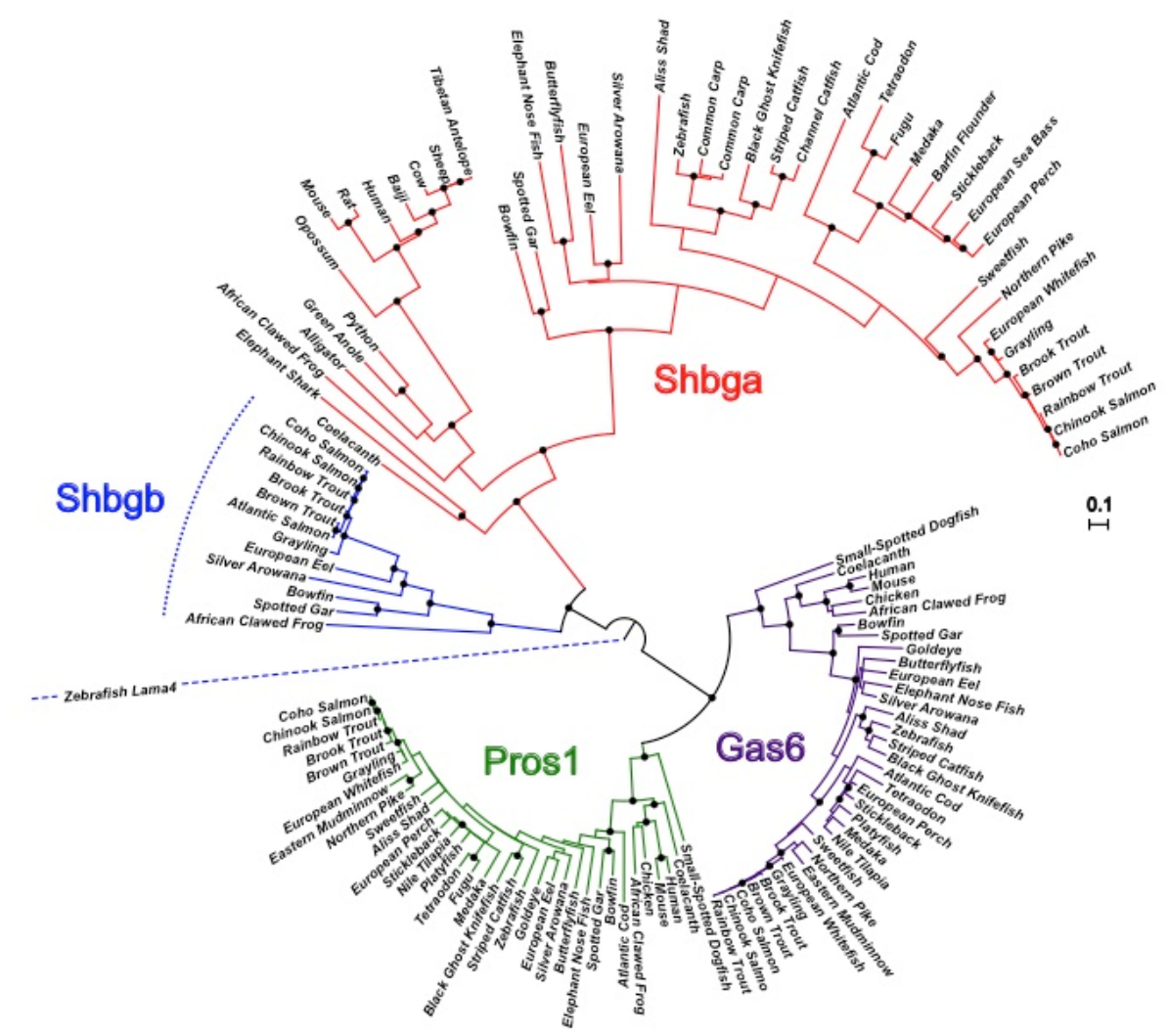

Figure 1: Phylogenetic reconstruction of the evolution of LamG domains proteins (Shbg, Gas6 and Pros1) in vertebrates. Circular NJM phylogenetic tree of LamG domains proteins including Shbga in red, Shbgb in blue, Gas6 in purple and Pros1 in green. The tree is rooted using zebrafish Laminin Subunit Alpha 4 (lama4) and bootstrap values over 0.75 are shown with a black dot on each significant node. 


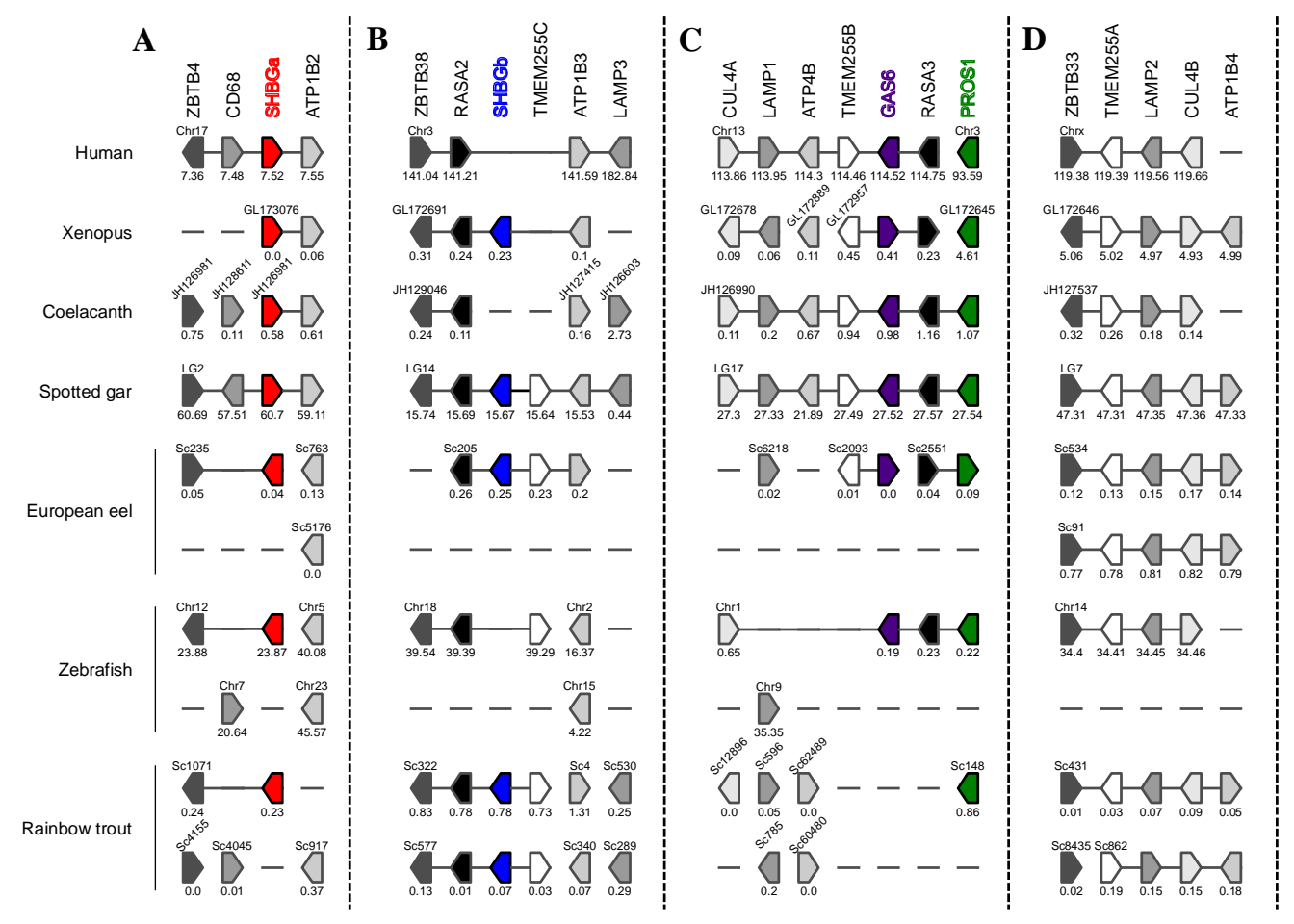

441 Figure 2: Synteny maps of conserved genomic regions around LamG domains 442 proteins (i.e. Shbga, Shbgb, Gas6 and Pros1) in human, Xenopus, coelacanth, spotted gar, Euroean eel, zebrafish and rainbow trout. Synteny maps are given for genomic regions around Shbga (A), Shbgb (B), Gas6 and Pros1 locus (C) and for a fourth region containing homologs of neighbouring Shbga, Shbgb, Gas6 and Pros1 genes (D). Genes are represented by blocks with an arrowed side indicating the gene orientation on chromosomes, linkage group or scaffolds. Gene location on chromosomes (Chr for Human and zebrafish), Linkage group (LG for spotted gar) and scaffolds (Ensembl reference or scaffold number) is

450 given in Mb below each gene block. Genes belonging to the same Chr, LG or scaffolds are 451 linked by a solid line. 

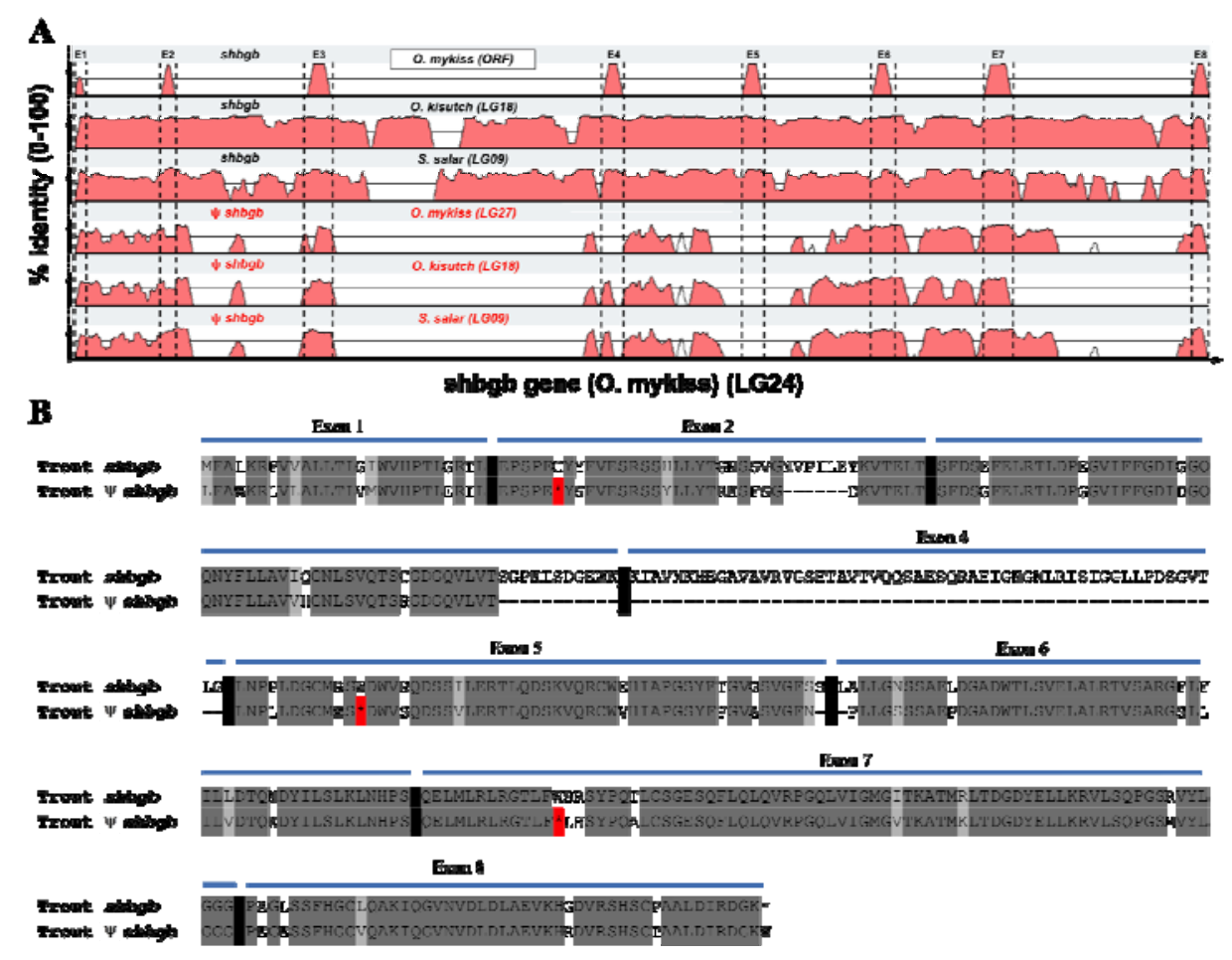

454 Figure 3: Multiple alignments plots of shbgb genes in salmonids. (A). Percentage of 455 sequence identity of shbgb genes in Atlantic salmon, Salmon salar, and Coho salmon, 456 Oncorhynchus kisutch compared to the rainbow trout, Oncorhynchus mykiss, shbgb gene on 457 linkage group (LG) 24. In addition to the functional shbgb genes that were found in all 458 salmonid species investigated, all these species have an additional shbgb homolog 459 containing multiple stop codons and thus considered as a pseudogene ( $\psi$ shbgb). (B). 460 Rainbow trout Shbgb and $\psi$ Shbgb protein alignment showing that the $\psi$ Shbgb contains 461 462 multiple stop codons (red asterisks) and a large deletion in exon 4 of the Shbgb protein. 

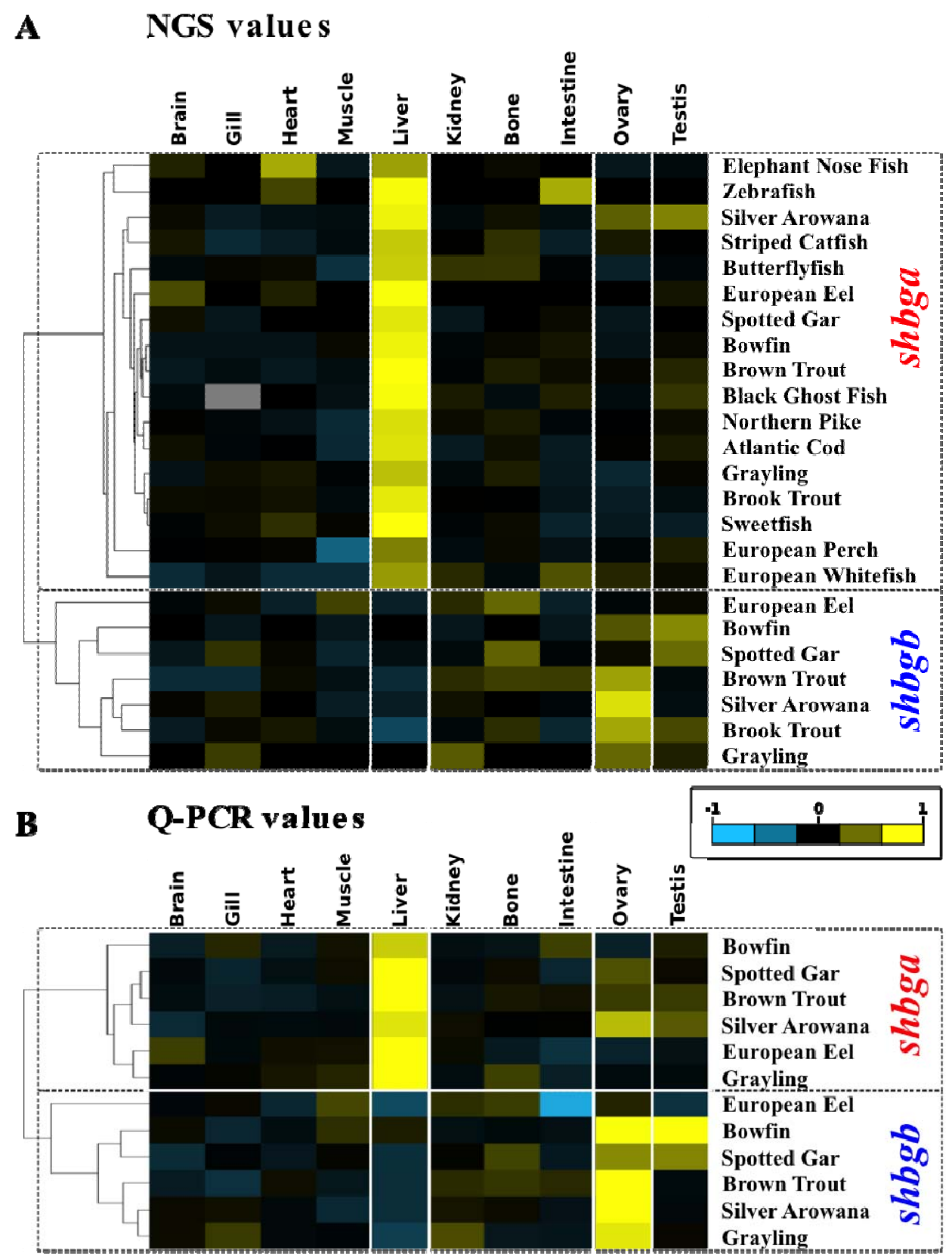

Figure 4: Tissue expression profiles of shhbga and shbgb genes. (A). Heatmap (colorized matrix) of the hierarchical clustering of tissue RNA-Seq expression profiles (NGS values) of shbg genes in different Holostean (spotted gar and bowfin) and teleost species. A predominant expression of shbga is found in the liver contrasting with the predominant expression of shbgb in gonads (B). Heatmap (colorized matrix) of the hierarchical clustering of tissue expression profiles of shbg genes analyzed by quantitative PCR (QPCR values) in different Holostean (spotted gar and bowfin) and teleost species. Colorized matrixes highlight the high expressing tissues in yellow, the low expressing tissues in blue and the median expression in black (see color scale). 


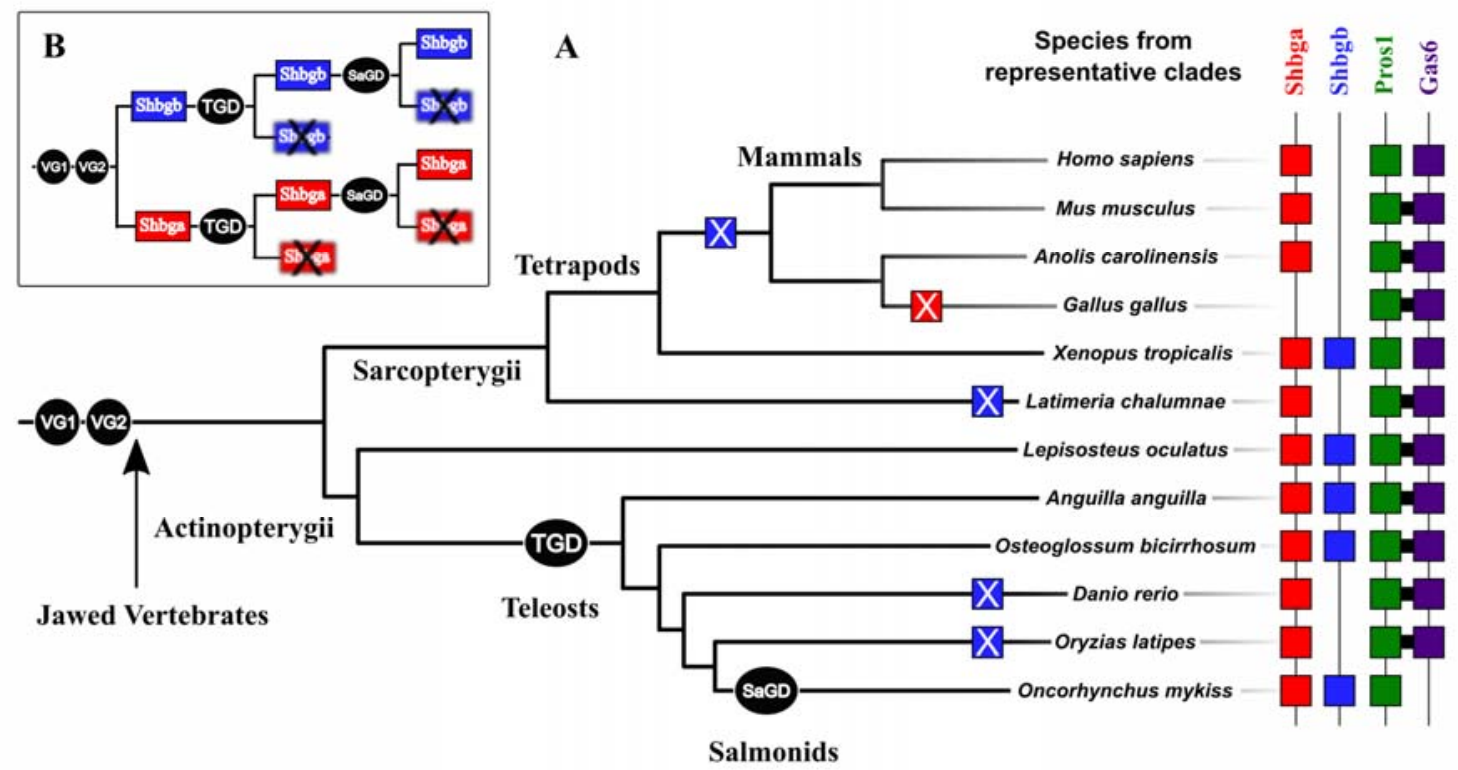

Figure 5: Evolution of Shbg/shbg, Gas6/gas6, and Pros1/pros1 genes through successive whole genome duplications and independent gene losses. (A). Schematic representation of the evolution of LamG domains proteins including, Shbga (red squares), Shbgb (blue squares), Gas6 (purple squares) and Pros1 (green squares) in some jawed vertebrates' representative species. Whole genome duplications (black circles; VG1 and VG2: vertebrate genome duplications 1 and 2, TGD: teleost genome duplication, SaGD: salmonid genome duplication) are indicated at each duplication nodes. Gene losses are represented by square boxes with a cross inside. (B). Simplified representation of the evolution of Shbg proteins after whole genome duplications showing the systematic losses of

490 one Shbga and Shbgb paralog after each duplication. 


\section{Supplementary Figures}
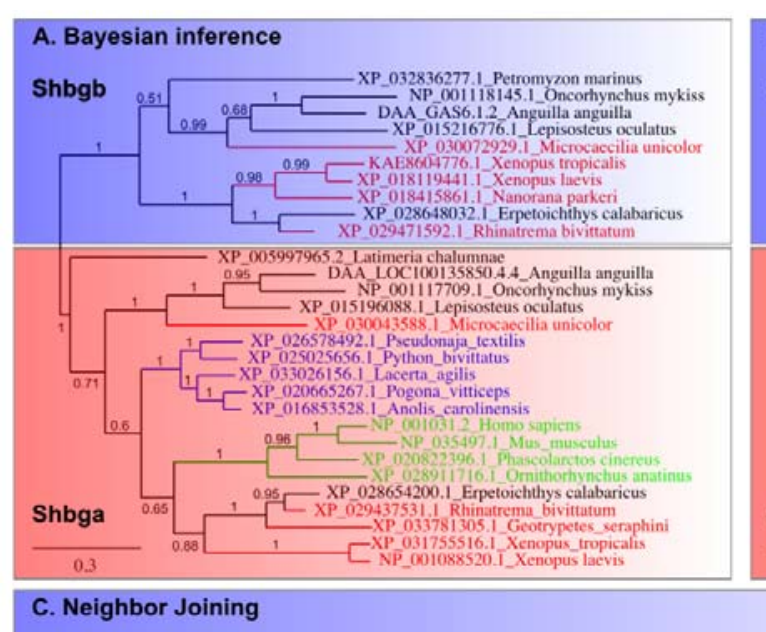

493

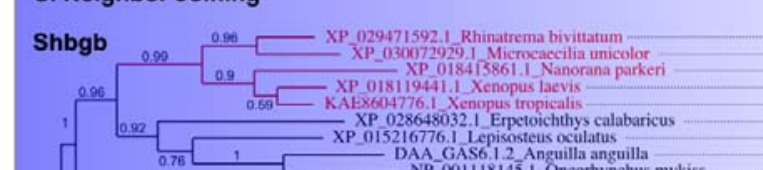

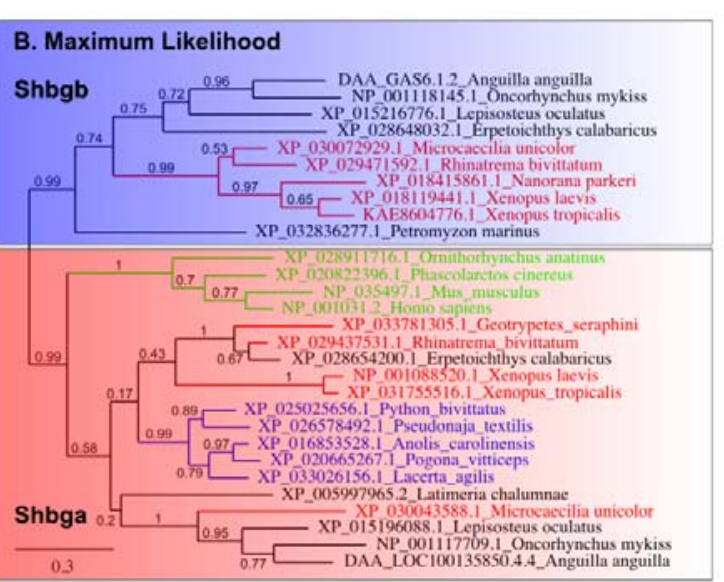

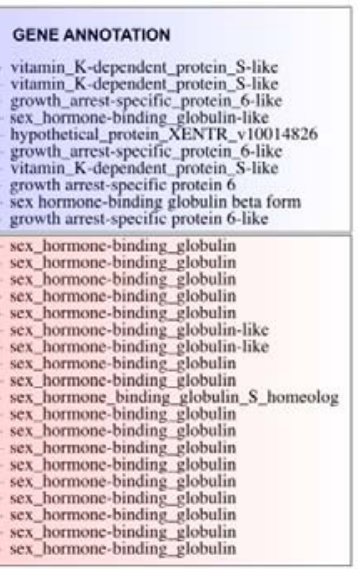

494 Supplementary Figure 1: Phylogenetic trees of some vertebrate Shbga and Shbgb

495 proteins. Protein sequences were searched in the ref-seq GenBank database using blastp 496 (protein-protein BLAST) and in the the PhyloFish website (http://phylofish.sigenae.org/) using 497 Shbga and Shbgb proteins from spotted gar (Lepisosteus oculatus, XP_015196088.1 and 498 XP_015216776.1) and Western claw frog (Xenopus tropicalis, XP_0311755516.1 and 499 KAE 8604776.1$)$ as baits. Phylogenetic analyses were performed on the Phylogeny.fr website 500 (http://www.phylogeny.fr). Sequences were aligned with MUSCLE (v3.8.31) and cleaned with 501 Gblocks (v0.91b) with default settings. Phylogenetic trees were reconstructed using the 502 bayesian inference (A.) method implemented in the MrBayes program (v3.2.6), the maximum 503 likelihood method (B.) implemented in the PhyML program (v3.1/3.0 aLRT) with 100 504 bootstrap replicates, and the neighbor joining method (C.) implemented in the BioNJ 505 program. All trees were reconstructed with default settings and the graphical representation 506 and edition of the phylogenetic tree were performed with TreeDyn (v198.3). Proteins and tree branches are depicted in blue for reptiles red for amphibians and in green for mammals. In panel C., the GenBank annotations are given for all protein sequences (with the exception of Shbg protein in Anguilla anguilla that were retrieved from the PhyloFish website), showing that all Shbga proteins are well annotated but that most Shbgb proteins are mis-annotated as Pros1-like or Gas6-like proteins. 


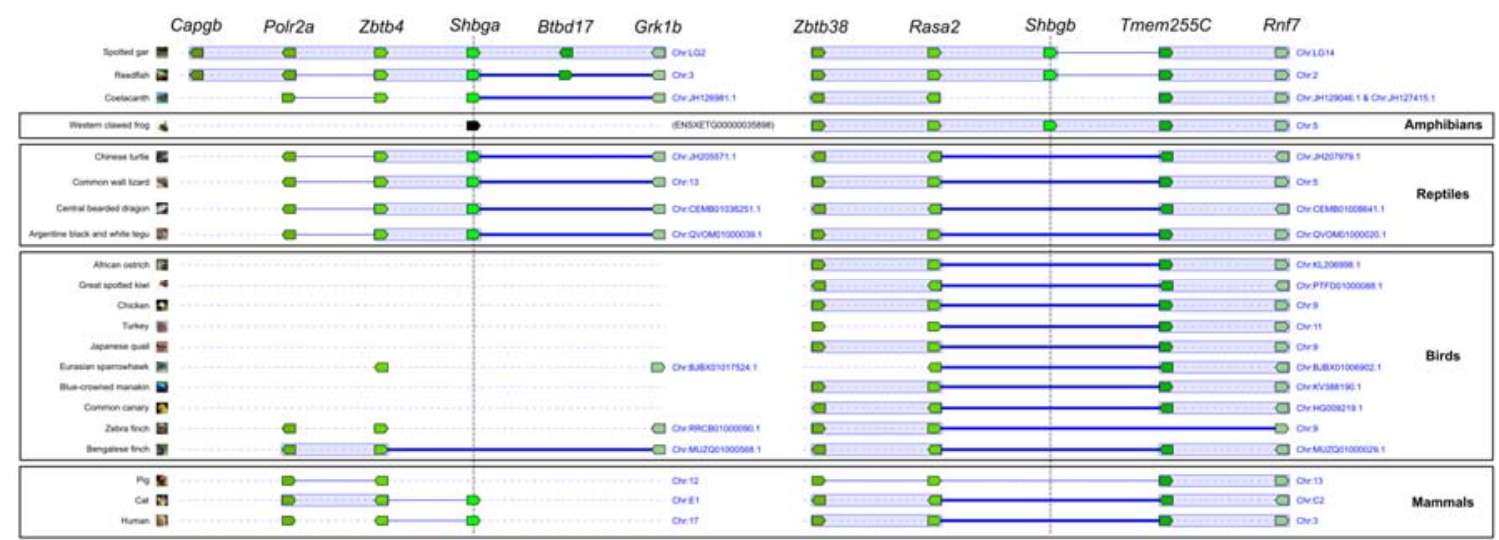

515 Supplementary Figure 2: Genomic context and synteny relationships of some 516 vertebrate Shbga and Shbgb proteins, showing the absence of both Shbga and Shbgb 517 in birds and of Shbgb only in mammals and reptiles. This analysis was performed using 518 the Genomicus genome browser (version 99.01, 519 https://www.genomicus.biologie.ens.fr/genomicus-99.01/) using as seed sequences the 520 accession numbers of Shbga (ENSLOCG00000013875) and Shbgb 521 (ENSLOCG00000008107) of spotted gar (Lepisosteus oculatus). Only a subset of species is depicted in this figure but the absence of both Shbga and Shbgb in birds and of Shbgb only in mammals and reptiles was consistently found in all birds $(\mathrm{N}=35)$, mammals $(\mathrm{N}=99)$ and reptiles $(\mathrm{N}=14)$ genomes available in the Genomicus version 99.01. No Shbg homolog were found in the elephant shark, hagfish and lamprey genomes available in this Genomicus version 99.01. 